\title{
Electronic band structure of carbon nanotube superlattices from first-principles calculations
}

\author{
A. Ayuela, ${ }^{1}$ L. Chico, ${ }^{2}$ and W. Jaskólski ${ }^{3}$ \\ ${ }^{1}$ Donostia International Physics Center (DIPC) and Unidad de Física de Materiales, Centro Mixto CSIC-UPV/EHU, 20080 Donostia, \\ Spain \\ ${ }^{2}$ Departamento de Física Aplicada, Facultad de Ciencias del Medio Ambiente, Universidad de Castilla-La Mancha, 45071 Toledo, Spain \\ ${ }^{3}$ Instytut Fizyki UMK, Grudziązka 5, 87-100 Toruń, Poland
}

(Received 4 August 2007; revised manuscript received 14 November 2007; published 29 February 2008)

\begin{abstract}
We report on first-principles calculations for metallic carbon nanotube superlattices $N(12,0) / N(6,6)$ with $N=1-4$. Although the calculated band structures show a good overall agreement with the results of the simpler tight-binding $\pi$-electron approximation, electron interaction and correlation effects strongly modify some peculiar flatbands, previously found within a tight-binding approach [W. Jaskólski and L. Chico, Phys. Rev. B 71, 155405 (2005)]. In the ab initio approach, these bands are no longer dispersionless, much closer to the Fermi level, and are always nondegenerate, in contrast to former tight-binding results.
\end{abstract}

DOI: 10.1103/PhysRevB.77.085435

PACS number(s): 73.22.-f, 73.21.Fg, 73.22.Dj, 71.15.Mb

\section{INTRODUCTION}

Since their discovery in $1991,{ }^{1}$ carbon nanotubes $(\mathrm{CNTs})$ are the subject of a growing interest due to their potential applications in nanoelectronic devices. As carbon nanotubes can be either metallic or semiconducting depending on their geometry, they stand as possible candidates for the design of all-carbon nanoelectronics. A wide variety of nanotube-based electronic devices, such as diodes, ${ }^{2,3}$ transistors, ${ }^{4}$ or field emitters, ${ }^{5}$ has been already theoretically modeled and experimentally achieved. Some of these applications rely on modifications of the electronic properties of carbon nanotubes caused by mechanical deformations ${ }^{6}$ or topological defects. By introducing topological defects into the hexagonal carbon network, the nanotube chirality may change and therefore its electronic characteristics. In this way, two-terminal elements, such as metallic-semiconductor junctions, ${ }^{7}$ quantum dots, ${ }^{8-11}$ or superlattices, ${ }^{12-14}$ can be designed. Carbon nanotube three- and four-terminal devices ${ }^{15}$ and networks ${ }^{16}$ have been also proposed in the same fashion, i.e., by joining nanotubes of different geometries via the introduction of topological defects. From the practical viewpoint, most simple nanotube devices ${ }^{2,4}$ have been found accidentally, but these pioneering works have stimulated research on their systematic production. In fact, quite recently, the controlled production of several carbon nanotube intramolecular junctions by temperature changes during growth ${ }^{17}$ has been reported. This finding may allow for the production of carbon nanotube quantum dots and superlattices in a controllable fashion, providing single-wall carbon nanotube nanoelectronic components.

On the theoretical side, much effort has been devoted toward the understanding of the properties of single junctions and quantum dots made of carbon nanotubes. A review of theoretical works devoted to carbon nanotube junctions can be found in Ref. 18. Completely localized states have been predicted in semiconductor-metal-semiconductor $(S / M / S)$ structures of diverse geometries ${ }^{8,9}$ All-metallic quantum dots have also been investigated; ${ }^{9-11}$ in some of these systems, it is possible to achieve completely localized states by exploring the existence of a symmetry gap between metallic tubes of different symmetries. ${ }^{11}$ Superlattices (SLs) made of car- bon nanotubes have not been so widely studied. Lu et al. ${ }^{13}$ studied the electronic and transport properties of metallic superlattices composed of zigzag nanotubes. Jaskólski and Chico $^{12,14,19}$ investigated metallic superlattices made of zigzag, armchair, and chiral tubes. In $N(2 n, 0) / M(n, n)$ superlattices, ${ }^{12}$ they found a series of peculiar dispersionless bands, some of them being multiply degenerate.

To date, the electronic structure of carbon nanotube junctions, quantum dots, and superlattices has been mainly studied within the tight-binding (TB) approach. The role of the electron interaction and correlation effects in these systems has not been investigated yet. The aim of this paper is to fill this gap and to explore the electronic properties of carbon nanotube $N(12,0) / N(6,6)$ superlattices by using the density functional theory (DFT) and including atomic structure relaxation. In principle, due to the high number of defects, strong interface strain and lattice relaxation effects are expected to change the band structure, especially for short unit cell superlattices. Furthermore, we investigate the role of electron interaction and correlation on the energy structure of superlattices. In particular, we study the influence of these effects on dispersionless bands, which deserve a more detailed study in order to find out to what extent their peculiar behavior is model-dependent. Dispersionless bands at the Fermi level are known to be responsible of interesting effects, such as spin polarization and the so-called flatband magnetism. Moreover, optical spectra will clearly show the signatures of these dispersionless bands due to their high density of states.

The paper is organized as follows. In Sec. II, we discuss the computational details. In Sec. III, we present our results for the relaxed structures and we compare them with previous tight-binding results. ${ }^{12}$ Identification of the origin of some superlattice bands is performed in Sec. IV.

\section{COMPUTATIONAL DETAILS}

We have performed DFT electronic structure calculations employing the SIESTA $a b$ initio code..$^{20}$ We work within the generalized gradient approximation for the exchangecorrelation effects, ${ }^{21}$ using the Troulliers-Martin norm- 


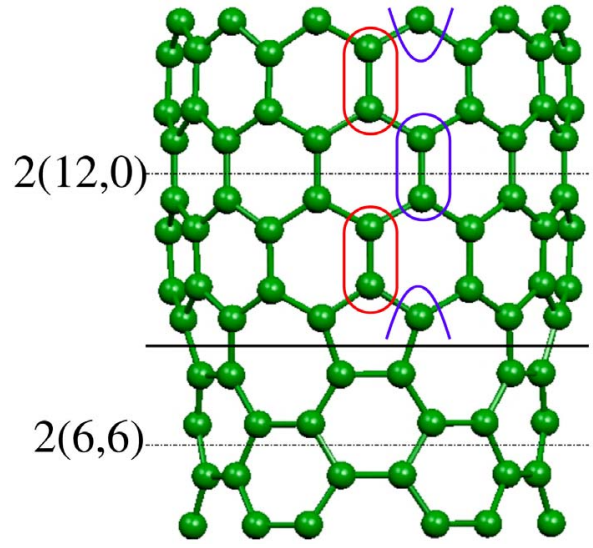

FIG. 1. (Color online). Schematic representation of a $(12,0) /(6,6)$ junction. Ovals represent the bean-shaped orbitals that give rise to the dispersionless bands in the $(12,0)$ section.

conserving pseudopotentials. Valence electrons are described with a double- $\xi$ singly polarized basis set. The cutoff radii for the $s$ and $p$ orbitals are 5.94 and 5.38 a.u., respectively. These radii have been optimized for diamond. In contrast to the basis optimized for graphite, the diamond basis set yields the carbon nanotube structures and total energies in better agreement with the results of standard plane-wave calculations; this improvement can be explained by the partial $s p^{3}$ hybridization shown by carbon in nanotubes. Real-space integration is performed on a regular grid corresponding to a plane-wave cutoff around $150 \mathrm{eV}$, which is usually enough to have converged calculations within several meV.

There are 48 atoms in the $(12,0)$ unit cell and 24 atoms in the $(6,6)$ unit cell; the atoms are grouped in four and two atomic layers, respectively. The nanotube (NT) superlattice is formed by introducing six pentagon/heptagon pairs at the $(12,0) /(6,6)$ interface; the $2(12,0) / 2(6,6)$ superlattice unit cell, which has 144 atoms, is plotted in Fig. 1. Then, isolated NT superlattices are modeled within the supercell approach. Perpendicular to their axis, the distances between NT superlattices are larger than $45 \AA$, so we can neglect their interaction. The input distance along the superlattice depends on $N$, i.e., on the size of the superlattice unit cell. We have calculated the theoretical lattice constant and have relaxed the coordinates until the forces are below $0.04 \mathrm{eV} / \AA$.

Special care has been given to the choice of the number of $k$ points in the band structure calculations. First, we tested the uniform infinite nanotubes. To achieve convergence down to $1 \mathrm{meV}$, the $(12,0)$ zigzag nanotube requires $20 k$ points, while for the $(6,6)$ armchair nanotube, $6 k$ points are needed. We focused our attention on the reproduction of the band structures and some of their peculiar features obtained with previous all-electron and pseudopotential calculations. $^{22,23}$ For the $(12,0)$ nanotube, we looked at the two bands at $k=0$, with the Fermi energy between them and the localized band around $-2.5 \mathrm{eV}$ below the Fermi level. For the $(6,6)$ nanotube, we considered the band crossing around $2 / 3$ of half the Brillouin zone, i.e., at the Fermi wave vector. To reproduce the previously calculated bands, ${ }^{22,23}$ the nanotubes have to be fully relaxed. Thereafter, performing the reciprocal lattice integrations for superlattices, we used variable number of $k$ points depending on the length of the superlattice unit cell. In the process of geometry optimization, the numbers of $k$ points used in the irreducible Brillouin zone are $8,8,5$, and 4 for $N=1,2,3$, and 4 superlattices, respectively. When plotting the band structures, we used larger number of $k$ points, i.e., 31, 15, 11, and 9 .

The results of $a b$ initio calculations are compared with the results of a tight-binding approximation. In this approach, we use one $\pi$ orbital per atom. The hopping parameter for nearest neighbors is fixed to $V_{p p \pi}=t=-2.66 \mathrm{eV}$; second-neighbor interactions are neglected.

\section{SUPERLATTICE BAND STRUCTURE}

In this section, we discuss the band structures of $N(12,0) / N(6,6)$ superlattices with $N=1-4$, paying particular attention to differences with respect to the tight-binding calculations. The detailed analysis of the so-called dispersionless bands, found within a tight-binding model, ${ }^{12}$ is included. In the left column of Fig. 2, we show the $a b$ initio band structure of the $N(12,0) / N(6,6)$ superlattices. The corresponding tight-binding results obtained within the $\pi$-electron approximation are presented in the right column. The Fermi level has been aligned in the corresponding pairs of subfigures. ${ }^{24}$ Surprisingly, in the tight-binding model, the superlattice Fermi level is not at zero energy, as it happens in case of uniform CNTs - where the Fermi level is at $0 \mathrm{eV}$ because of electron-hole symmetry-or in case of CNT quantum dots connected with CNT metallic leads, ${ }^{11}$ where the outer leads act as electron reservoirs, fixing the Fermi level at $0 \mathrm{eV}$. The shift of the Fermi level in superlattices can be ascribed to the appearance of bands that are asymmetric with respect to zero energy, i.e., to the center of the energy spectrum of a perfect CNT. The presence of these bands is due to the pentagon-heptagon interface defects, which are known to break the electron-hole symmetry. Furthermore, these asymmetric bands lie below $E=0 \mathrm{eV}$, shifting down the Fermi energy in CNT superlattices. Figure 2 shows also that the position of $E_{F}$ relative to some analogous bands of $a b$ initio and tight-binding calculations is very similar in both approaches.

It is widely known that tight-binding models give an adequate description of the nanotube band structure around $E_{F}$ for infinite perfect tubes. ${ }^{22}$ Figure 2 reveals that this is also true for $N(12,0) / N(6,6)$ CNT superlattices. In an overall comparison, the $a b$ initio bands agree well with the tightbinding picture, especially when focusing on dispersive bands. Flatbands corresponding to the so-called dispersionless (DL) bands of the tight-binding model ${ }^{12}$ are also present in the $a b$ initio calculations; in Fig. 2, they are indicated by arrows. They are broadened, their energies are significantly changed, and they are no longer dispersionless, but they are still narrower than the other bands. We will call them "weakly dispersive" (WD) bands, keeping the term "dispersionless" (DL) for their tight-binding $k$-independent counterparts.

To understand why DL bands become dispersive in the $a b$ initio approach, notice that DL bands at $\pm t$ are also present 
(a) $1(12,0) / 1(6,6)$
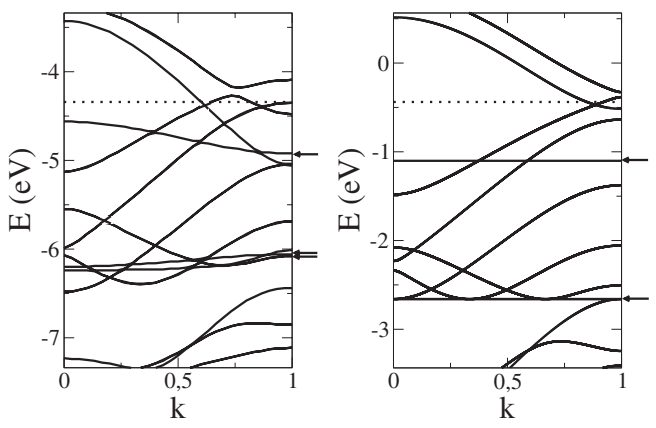

(b) $2(12,0) / 2(6,6)$
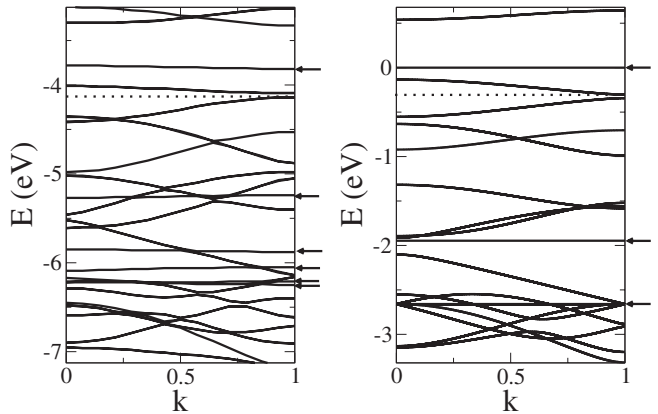

(c) $3(12,0) / 3(6,6)$
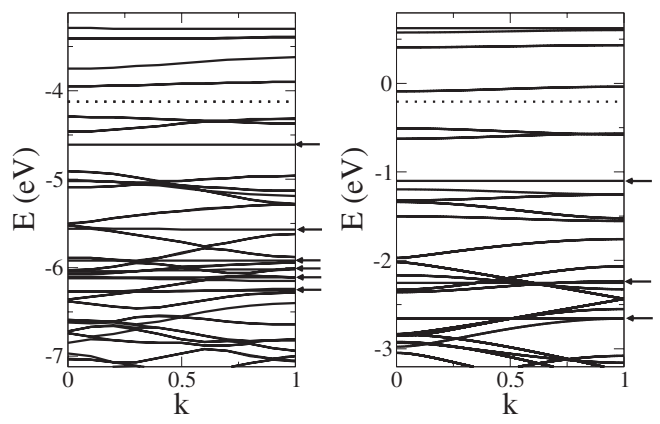

(d) $4(12,0) / 4(6,6)$
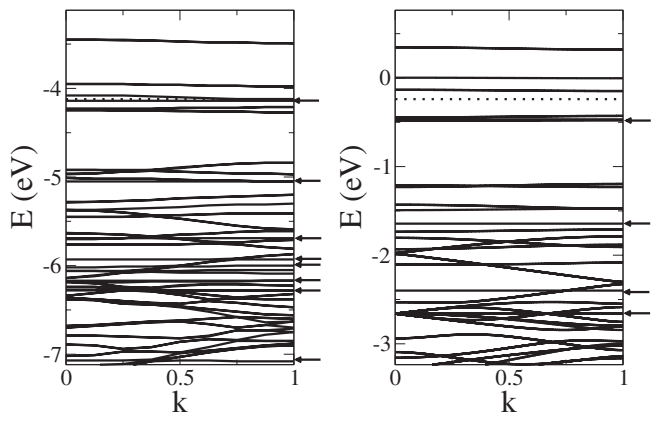

FIG. 2. Band structure for the $N(12,0) / N(6,6)$ superlattices around and below $E_{F}$ for (a) $N=1$, (b) $N=2$, (c) $N=3$, and (d) $N$ =4. $A b$ initio calculations, left panel; tight-binding results, right panel. The Fermi levels for $a b$ initio (tight-binding) calculations are $-4.34(-0.44) \mathrm{eV},-4.13(-0.32) \mathrm{eV},-4.12(-0.20) \mathrm{eV}$, and $-4.12(-0.24) \mathrm{eV}$ following the (a)-(d) order. Dispersionless and the corresponding weakly dispersive bands are indicated by arrows. The wave vector $k$ is given in reciprocal space units. The superlattice unit cell lengths $a_{S L}$ are $6.25 \AA$ for $N=1,13.54 \AA$ for $N=2$, $20.29 \AA$ for $N=3$, and $27.04 \AA$ for $N=4$. in infinite $(2 n, 0)$ CNTs, even in a four-orbital tight-binding calculation including curvature effects. On the other hand, WD bands appear in $a b$ initio calculations without structural relaxation, so we attribute the appearance of weak dispersion to the electron correlation. The degeneracy of DL bands appearing at $E= \pm t$ in the tight-binding model is always lifted in the $a b$ initio calculations. Most remarkably, while the DL bands are strongly modified by the electron interaction, the dispersive ones are much less affected, as it can be seen by comparing the right and left panels in Fig. 2. In all cases, WD bands are closer to the Fermi level than their DL counterparts. This may imply that, by slightly doping the superlattices, flatbands could show spin polarization due to the large density of states at the Fermi level, so that the Stoner criterion would be fulfilled.

\section{LOCALIZATION PROPERTIES OF DISPERSIONLESS AND WEAKLY DISPERSIVE BANDS}

Since DL bands are the most affected by electron-electron interaction, we discuss their localization properties in more detail. As shown in Ref. 12, a series of DL bands appear in the tight-binding $\pi$-electron approximation for all $N(12,0) / M(6,6)$ superlattices. There are always degenerate bands at $E= \pm t$. Their degeneracy amounts to $2 N$, where $N$ is the number of $(12,0)$ unit cells in the superlattice unit cell, as indicated above. These degenerate bands can be traced back to the dispersionless bands of the $(12,0)$ nanotube that occur at the same energies (see Fig. 3). $2 N-1$ of these degenerate bands are localized in the internal atomic layers of the $N(12,0)$ part of the superlattice unit cell; the remaining one is localized in the $N(6,6)$ section and extends to the outermost atomic layers of the $N(12,0)$ section. Another dispersionless bands which lie at energies $E \neq \pm t$ are always nondegenerate; they are localized in the $M(6,6)$ part and extend up to the adjacent atomic layers of the $N(12,0)$ section. The number of these bands equals to $M$, where $M$ is the number of the $(6,6)$ unit cells in the superlattice unit cell. ${ }^{25}$ Some of these DL bands are shown in the left panels of Fig. 2, marked with arrows.

A comparison of localization properties of some DL bands and their WD counterparts, for $1(12,0) / 1(6,6), 2(12,0) / 2(6$, $6)$, and $3(12,0) / 3(6,6)$ superlattices, is presented in Figs. 4 and 5. Only DL and WD bands with energies $E \leqslant E_{F}$ are shown, with the exception of one localized band in the $N$ $=M=2$ case appearing just above the Fermi level. Wave functions of the WD bands are depicted in the left panels; the tight-binding eigenvectors of the corresponding DL states are represented in the right panels. Each component of any TB eigenvector is represented by a sphere of radius proportional to its modulus; different signs of the wave function are represented by different colors. ${ }^{26}$ All wave functions are labeled by their band energies in $\mathrm{eV}$ at $k=0$.

Let us consider first the WD bands which form degenerate multiplets at $E=-2.66 \mathrm{eV}$ in the TB model. In the case of $1(12,0) / 1(6,6) \mathrm{SL}$, the corresponding wave functions are shown in the two lowest pairs of pictures of Fig. 4(a), labeled -6.24 and -6.20 in the $a b$ initio approach. Although these bands are nondegenerate in the ab initio calculations (their 

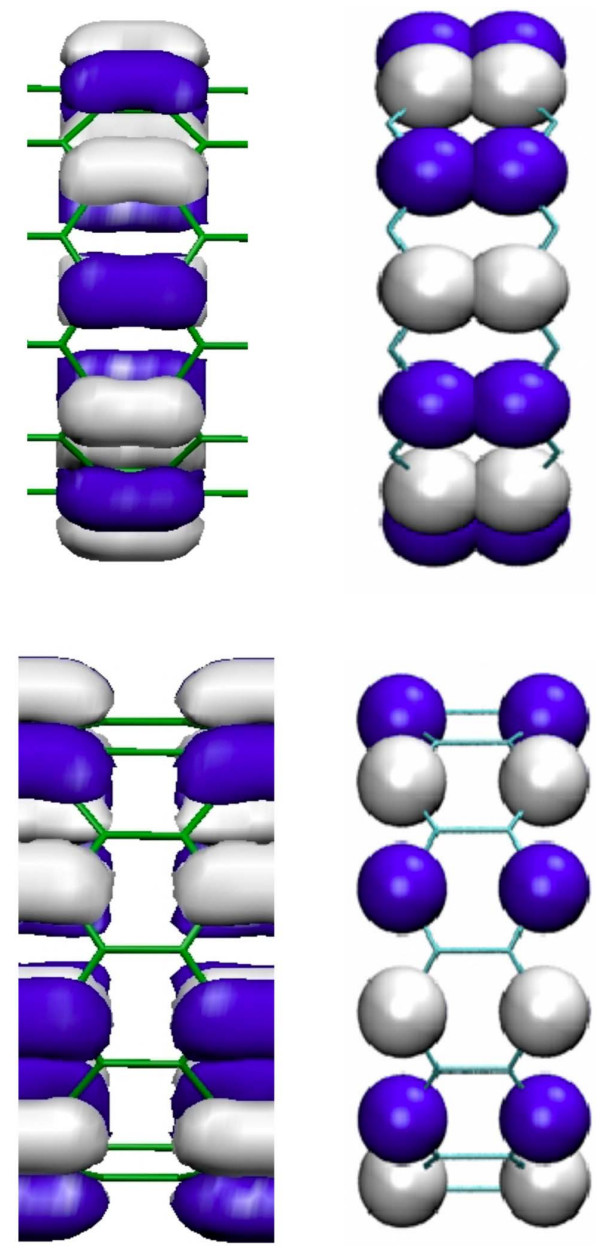

FIG. 3. (Color online) Orbitals of the WD (left) and DL (right) bands at $k=0$ for the $(12,0) \mathrm{CNT}$ around $-2.66 \mathrm{eV}$ below the Fermi energy (see caption of Fig. 4 for details).

energy splitting at $\Gamma$ is $0.04 \mathrm{eV}$ ), their wave functions are very similar to the ones resulting from the $\mathrm{TB}$ calculations. The lower energy state is almost exclusively localized in the $(12,0)$ section. It stems from the bean-shaped orbitals between two carbon atoms, which are characteristic of degenerate DL bands at $E=-2.66 \mathrm{eV}$ of the perfect $(12,0)$ nanotube (see Fig. 3). This is the bonding state having the localized Kekule double bonds along the axial direction of the zigzag tube, ${ }^{27}$ which are allowed by the finite zigzag topology but not by the finite armchair one.

Similar states are also present in case of $2(12,0) / 2(6,6)$ and $3(12,0) / 3(6,6)$ superlattices [the two lowest $a b$ initio wave functions in Fig. 4(b), labeled -6.17 and -6.09 , and the two lowest in Fig. 5, labeled -6.10 and -6.04 (Ref. 28)]. For such states, the closer the beans are to the center of the $(12,0)$ section, the lower is the corresponding energy level. At $k=0$, these bands can also be strongly mixed with the low lying dispersive bands, seen already in the -6.17 WD band of Fig. 4. Other higher-energy states may have nodal planes perpendicular to the nanotube axis.

All the other DL bands and the corresponding WD bands have a predominant $(6,6)$ character; they are localized in the $N(6,6)$ part and extend to the two adjacent atomic layers of (a) $1(12,0) / 1(6,6)$

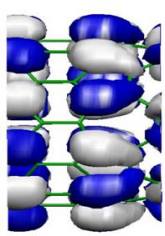

$-4.56$

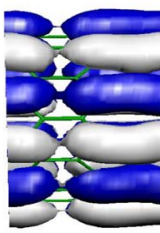

$-6.20$

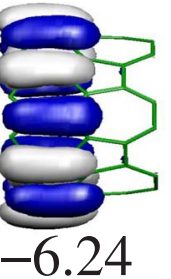

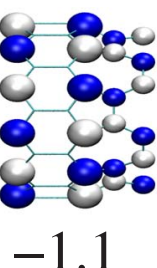
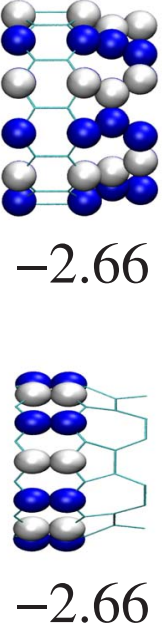

(b) $2(12,0) / 2(6,6)$
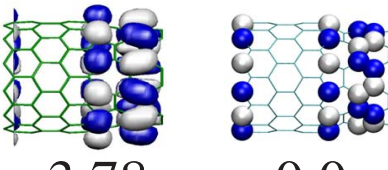

$-3.78$

0.0
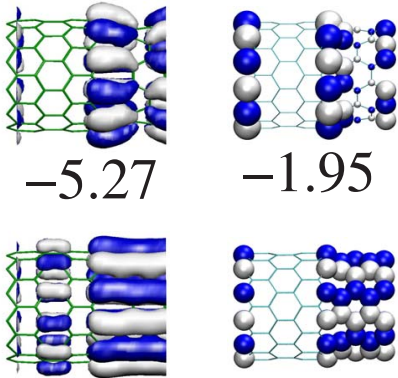

$-5.85$
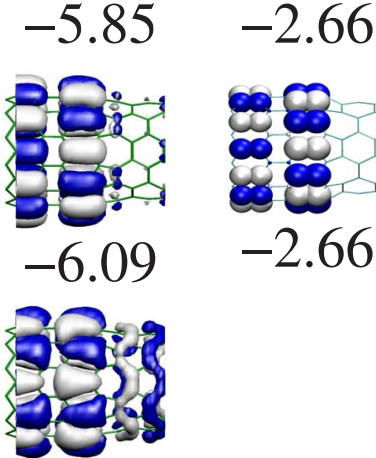

$-6.17$

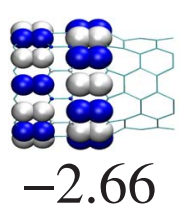

FIG. 4. (Color online) Some examples of WD (left panels) and dispersionless orbitals (right panels) around the Fermi energy, for superlattices with (a) $N=1$ and (b) $N=2$. WD orbitals are given in order of decreasing energies from top to bottom. The ab initio orbitals are cut at $10 \%$ of their maximum value. Positive lobes are shown in white (light gray), and negative ones in blue (dark). Likewise, each tight-binding eigenvector is represented by a sphere of radius proportional to its modulus; positive values are in white and negative values in blue (dark). Orbitals are labeled by their band energies in $\mathrm{eV}$ at $k=0$.

the $N(12,0)$ section. They are presented in the upper pairs of pictures in Figs. 4 and 5. In the tight-binding model, one of these bands belongs to the degenerate multiplet at $E=-2.66 \mathrm{eV}$; in the $a b$ initio calculations, this band splits, as the other WD bands belonging to this multiplet do. The remaining DL and WD bands have higher energies and are nondegenerate in both approaches. The main difference between the WD and DL states is that WD states have a mixed character, i.e., even if they are mainly localized in one section $[N(12,0)$ or $N(6,6)]$ of the SL unit cell, they are also weakly present in the other one.

A comment is required on the origin of the superlattice DL (or WD) bands. ${ }^{25}$ As mentioned above, the DL (or WD) bands with a predominant localization in the $N(12,0)$ sections originate from the corresponding DL bands of the perfect $(12,0)$ tube (see Fig. 3). The remaining WD bands that are mainly localized in the $(6,6)$ section should be analyzed 


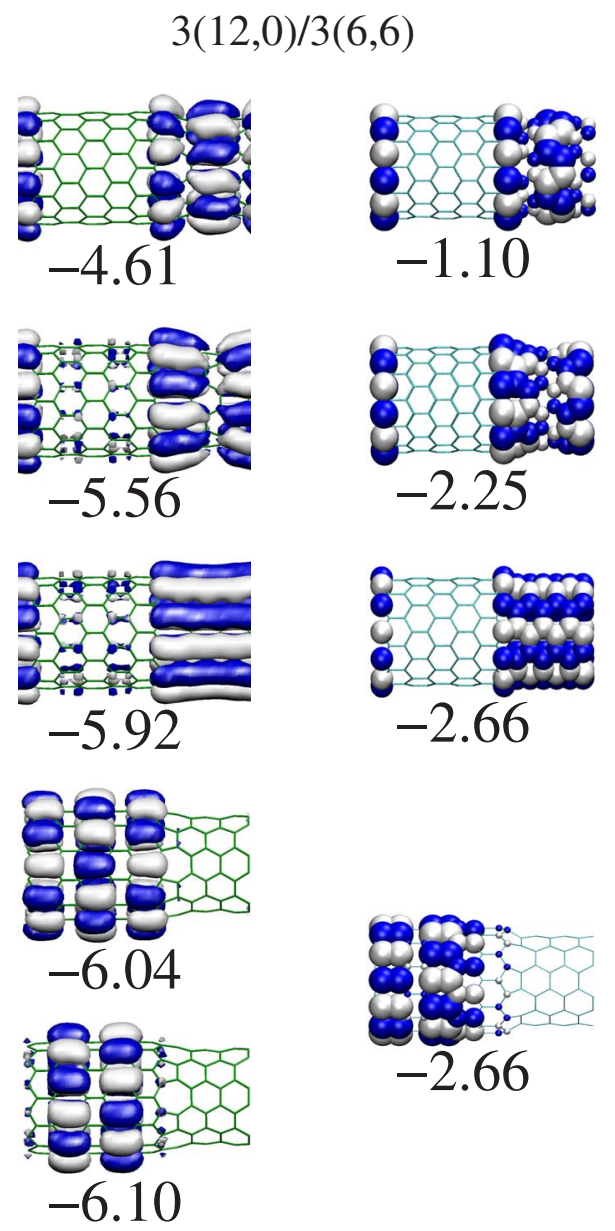

FIG. 5. (Color online) Some examples of WD (left panels) and dispersionless orbitals (right panels) around the Fermi energy for the $3(6,6) / 3(12,0)$ superlattice. WD orbitals are given in order of decreasing energies from top to bottom (see caption of Fig. 4 for details).

in connection with the bands of infinite $(6,6)$ NT. Let us therefore focus on the band of the $(6,6)$ tube which for $k$ $=0$ begins at $-2.66 \mathrm{eV}$ (following Ref. 14, we will call this band $B 6)$. This band is invariant under $C_{6}$ rotations. The inspection of its TB wave function (e.g., for $k=0$ ) reveals that for each atomic layer of the $(6,6)$ unit cell, it gets \pm 1 values at consecutive atoms of a given layer. The only bands of the $(12,0)$ tube having the same property are the DL bands at $E= \pm 2.66 \mathrm{eV}$. Therefore, when the SL is formed from the finite sections of the $(6,6)$ tube and when the abovementioned $B 6$ band quantizes, the resulting $(6,6)$-discrete states cannot tunnel through the $N(12,0)$ section since the corresponding band in the $(12,0)$ tube is dispersionless. Consequently, the discrete states arising from quantization of band $B 6$ extend only to the adjacent atomic layers of the $N(12,0)$ section and vanish in its internal part; this behavior fits to a quantum well picture. The wave functions of these B6-originated DL bands remind of quantum well states localized in the $N(6,6)$ section: the higher the energy, the larger the number of nodal planes perpendicular to the tube axis. This is especially well seen in case of $3(12,0) / 3(6,6)$ SL (see Fig. 5). In fact, the energies of these DL bands can
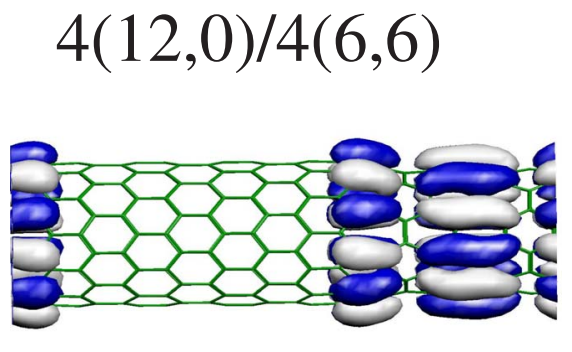
$-5.05$
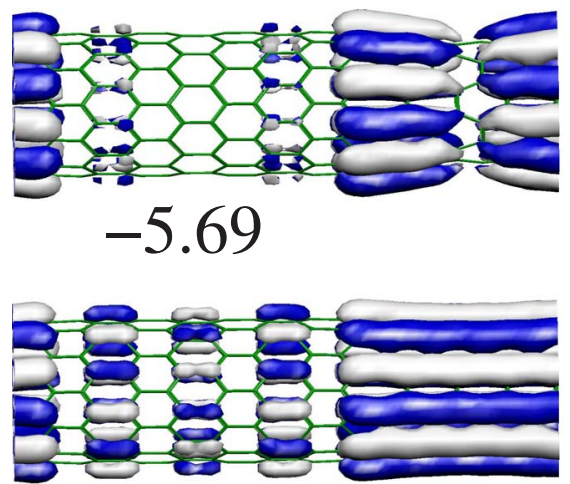
$-5.93$

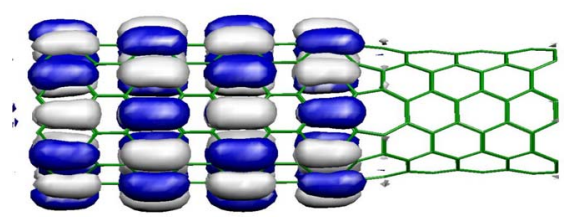

$-6.02$

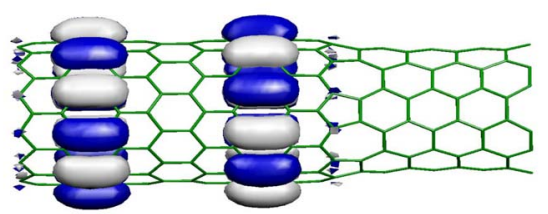

$-6.17$

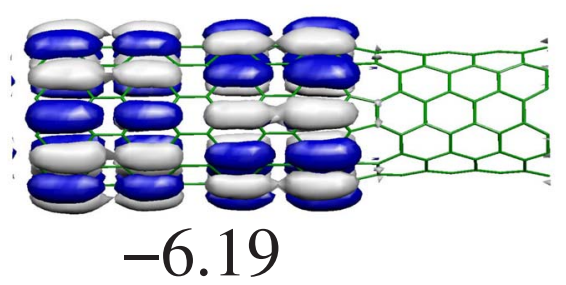

FIG. 6. (Color online) Some $a b$ initio WD orbitals around the Fermi energy for the $4(6,6) / 4(12,0)$ superlattice, shown in order of decreasing energies from top to bottom. The $a b$ initio orbitals are cut at $10 \%$ of their maximum value. Positive lobes are in white (light gray), and negative ones in blue (dark).

be found by zone folding, i.e., by sampling the $B 6$ band at $N$ evenly spaced $k$ points: In the $\pi$-electron tight-binding approximation, the $n$th band of the $(n, n)$ tube extends from $+t$ to $-t(t=-2.66 \mathrm{eV})$ and has the following energy dispersion: 


$$
E_{n}(k)=t\left[2 \cos \left(\frac{k a}{2}\right)-1\right],
$$

where $0<k<\frac{\pi}{a}$ and $a$ is the modulus of the graphene basis vectors. For the $(6,6)$ case, this is the $B 6$ band which crosses the Fermi level. One can easily check that for $N=1$, there is only one such state at $E=-1.1018 \mathrm{eV}$; for $N=2$, there are two states at $E=1.947 \mathrm{eV}$ and $E=0$, and so on. These values are exactly the energies of DL bands.

Regarding the WD bands of $B 6$ origin (being the counterparts of the quoted DL bands), they obviously have a predominant $(6,6)$ character, but their wave functions are no longer required to vanish in the internal part of the $(12,0)$ section. The lowest of these WD bands extends more substantially to the $(12,0)$ part. In the TB approach, its energy is always $-2.66 \mathrm{eV}$, independent on $N$, while in the $a b$ initio calculations, the energy of the corresponding WD orbital depends on the SL size: it is at $-6.20 \mathrm{eV}$ for $N=1,-5.85 \mathrm{eV}$ for $N=2$, and $-5.92 \mathrm{eV}$ for $N=3$. In each case, this state can be easily identified by the long shape of the orbital, extending along the $(6,6)$ section of the superlattice (see Figs. 4-6). This is a hybrid state made of the $B 6$ band of the $(6,6)$ tube and one of the DL (WD) of the $(12,0)$ tube; the shape of this $(12,0)$ wave function can be seen in the left parts of Fig. 3. It can be easily seen that the shape of these $(12,0)$ orbital matches perfectly the lowest quantized $B 6$ band of the $(6,6)$ tube.

Figure 6 gives the wave functions of some WD bands obtained by $a b$ initio calculations for the case of $4(12,0) / 4(6,6) \mathrm{SL}$. We present these states to assess the double bond character along the tube axis of the low lying WD states and to show that also for this SL $(N=4)$, these states have a quantum well character. As before, they are localized in the $(6,6)$ section but they decay into the $(12,0)$ part.

In conclusion, although the $E(k)$ dependence of localized bands is more strongly affected by electron interaction, the wave functions of these WD bands are very similar to their DL counterparts. At variance with DL bands, which localize exactly in one or another section of the SL unit cell, local- ization of WD bands is not perfect: they have a nonnegligible decay in the regions where their DL counterparts vanish exactly.

\section{SUMMARY}

We have performed first-principles electronic band structure calculations of $N(12,0) / N(6,6)$ carbon nanotube superlattices, with $N=1-4$, and compared our results with those obtained in a $\pi$-electron tight-binding approximation. We have shown a good overall agreement of the superlattice band structure calculated in both approaches. However, some peculiar flatbands found in the tight-binding approach which are degenerate in energy turn out to be weakly dispersive and no longer degenerate. Furthermore, their energies are strongly affected by electron interaction: in the $a b$ initio approach, they are closer to the Fermi energy, so they could exhibit flatband magnetism if the superlattices are doped. In spite of these differences, their wave functions are quite similar in both approaches, although they are not so localized in the first-principles calculation. Through a detailed comparison of $a b$ initio and tight-binding results, we have clarified the origin of these localized bands.

Finally, we have also found that in the tight-binding scheme with only one $\pi$-orbital per atom, the superlattice Fermi energy is not at $0 \mathrm{eV}$. We have shown that this effect is due to the existence of pentagon-heptagon interface effects, which break the electron-hole symmetry and lower the energies on the valence band.

\section{ACKNOWLEDGMENTS}

This work was supported by the ETORTEK (NANOMAT) program of the Basque government, the Junta de Comunidades de Castilla-La Mancha Grant No. PAI-05-047, Spanish MEC Grants Nos. FIS2004-06490-C03-00 and MAT2006-06242, and the European Network of Excellence NANOQUANTA NM4-CT-2004-500198. L.C. and W.J. gratefully acknowledge the DIPC for hospitality. D. Sánchez-Portal and J. Aizpurua are acknowledged for discussions.
${ }^{1}$ S. Iijima, Nature (London) 354, 56 (1991).

${ }^{2}$ P. G. Collins, A. Zettl, H. Bando, A. Thess, and R. E. Smalley, Science 278, 100 (1997).

${ }^{3}$ J. U. Lee, P. P. Gipp, and G. M. Heller, Appl. Phys. Lett. 85, 145 (2004).

${ }^{4}$ S. J. Tans, A. R. M. Verschueren, and C. Dekker, Nature (London) 393, 49 (1998).

${ }^{5}$ S. Fan, M. G. Chapline, N. R. Franklin, T. W. Tombler, A. M. Cassell, and H. Dai, Science 283, 512 (1999); K. A. Dean, P. von Allmen, and B. R. Cahalarnala, J. Vac. Sci. Technol. B 17, 1959 (1999).

${ }^{6}$ H.-S. Sim, C.-J. Park, and K. J. Chang, Phys. Rev. B 63, 073402 (2001).

${ }^{7}$ L. Chico, V. H. Crespi, L. X. Benedict, S. G. Louie, and M. L.
Cohen, Phys. Rev. Lett. 76, 971 (1996); L. Chico, L. X. Benedict, S. G. Louie, and M. L. Cohen, Phys. Rev. B 54, 2600 (1996); R. Saito, G. Dresselhaus, and M. S. Dresselhaus, ibid. 53, 2044 (1996); J. C. Charlier, T. W. Ebbesen, and Ph. Lambin, Phys. Rev. B 53, 11108 (1996).

${ }^{8}$ L. Chico, M. P. López Sancho, and M. C. Muñoz, Phys. Rev. Lett. 81, 1278 (1998)

${ }^{9}$ C. G. Rocha, T. G. Dargam, and A. Latgé, Phys. Rev. B 65, 165431 (2002); W. Zhang, W. Lu, and E. G. Wang, Phys. Rev. B 72, 075438 (2005).

${ }^{10}$ E. Jódar, A. Pérez-Garrido, and A. Díaz-Sánchez, Phys. Rev. B 73, 205403 (2006).

${ }^{11}$ L. Chico and W. Jaskólski, Phys. Rev. B 69, 085406 (2004).

${ }^{12}$ W. Jaskólski and L. Chico, Phys. Rev. B 71, 155405 (2005). 
${ }^{13}$ W. Lu, E. G. Wang, and H. Guo, Phys. Rev. B 68, 075407 (2003).

${ }^{14}$ W. Jaskólski, S. Stachów, and L. Chico, Acta Phys. Pol. A 108, 697 (2005).

${ }^{15}$ M. Menon and D. Srivastava, Phys. Rev. Lett. 79, 4453 (1997); A. Pérez-Garrido and A. Urbina, Carbon 40, 1227 (2002); D. Grimm, R. B. Muniz, and A. Latgé, Phys. Rev. B 68, 193407 (2003).

${ }^{16}$ J. M. Romero-Herrera, M. Terrones, H. Terrones, S. Dag, and V. Meunier, Nano Lett. 7, 570 (2007).

${ }^{17}$ Y. Yao, Q. Li, J. Zhang, R. Liu, L. Jiao, Y. T. Zhu, and Z. Liu, Nat. Mater. 6, 283 (2007).

${ }^{18}$ J-C. Charlier, X. Blase, and S. Roche, Rev. Mod. Phys. 79, 677 (2007).

${ }^{19}$ W. Jaskólski and L. Chico, J. Phys.: Conf. Ser. 30, 230 (2006).

${ }^{20}$ J. M. Soler, E. Artacho, J. D. Gale, A. García, J. Junquera, P. Ordejón, and D. Sánchez-Portal, J. Phys.: Condens. Matter 14, 2745 (2002).

${ }^{21}$ J. P. Perdew, K. Burke, and M. Ernzerhof, Phys. Rev. Lett. 77, 3865 (1996).

${ }^{22}$ S. Reich, C. Thomsen, and P. Ordejón, Phys. Rev. B 65, 155411
(2002).

${ }^{23}$ J. Mintmire and C. T. White, Appl. Phys. A 67, 65 (1998).

${ }^{24}$ In $a b$ initio calculations, the energies are measured relative to the vacuum level $\left(E_{v a c}=0\right)$, while in the tight-binding approach, the zero-energy level is determined by the on-site parameter, set equal zero.

${ }^{25}$ In Ref. 12, it was suggested that all the superlattice DL bands originate from the dispersionless bands of the $(12,0)$ tube. This is true only for DL bands at $E= \pm t$.

${ }^{26}$ In the case of $2(12,0) / 2(6,6) \mathrm{SL}$, the DL eigenvectors for $E$ $= \pm t$ are taken at $k \neq \Gamma$ and the spheres represent the real part of the eigenvectors. This is because these bands are degenerate at $\Gamma$ with other dispersive bands.

${ }^{27}$ G. Treboux, P. Lapstun, and K. Silverbrook, Chem. Phys. Lett. 302, 60 (1999).

${ }^{28}$ In the case of $3(12,0) / 3(6,6)$ SL, only one of the three DL wave functions, which localize in the $(12,0)$ section, is shown. This is because the degenerate DL states are strongly mixed and therefore their wave functions do not remind the wave functions of the corresponding WD bands. 